\title{
Thoracic paravertebral block versus transversus abdominis plane block in major gynecological surgery: a prospective, randomized, controlled, observer-blinded study
}

This article was published in the following Dove Press journal:

Local and Regional Anesthesia

I October 2012

Number of times this article has been viewed

\author{
Andrey L Melnikov' \\ Steinar Bjoergo' \\ Ulf E Kongsgaard ${ }^{2}$ \\ 'Department of Anesthesiology, \\ Division of Emergencies and Critical \\ Care, Oslo University Hospital, \\ Oslo, Norway; ${ }^{2}$ Department of \\ Research and Development, Division \\ of Emergencies and Critical Care, \\ Oslo University Hospital and Medical \\ Faculty, University of Oslo, Oslo, \\ Norway
}

Background and objectives: Patients undergoing abdominal surgery often receive an epidural infusion for postoperative analgesia. However, when epidural analgesia is contraindicated or unwanted, the administration of opioids is the usual means used to relieve pain. Various regional analgesia techniques used in conjunction with systemic analgesia have been reported to reduce the cumulative postoperative opioid consumption and opioid-induced side effects. The objective of this trial was to assess the effectiveness of transversus abdominis plane block and paravertebral block in women undergoing major gynecological surgery.

Methods: We analyzed 58 patients scheduled for a midline vertical laparatomy due to gynecological cancer. They were all equipped with a patient-controlled postoperative analgesia pump that delivered ketobemidon. In addition, some patients were randomized to receive either a bilateral transversus abdominis plane block $(n=19)$ or a bilateral paravertebral block at the level of Th10 $(\mathrm{n}=19)$. Both blocks were performed preoperatively as a single injection of bupivacaine.

Results: Cumulative ketobemidon consumption, postoperative pain scores at rest and while coughing, and postoperative nausea and vomiting scores were assessed by a blinded observer at $2,4,6,24$, and 48 hours postoperatively. Both blocks were associated with significant reductions in opioid consumption and pain scores throughout the study period compared with the control patients. Postoperative nausea and vomiting scores were low in all groups, but during the early postoperative period more control group patients needed antiemetics.

Conclusion: Both methods of inducing block can serve as effective analgesia adjuncts in women undergoing major gynecological surgery. Although thoracic paravertebral block appeared to be more effective than transversus abdomins block, the latter performed under ultrasound guidance seems to be a more controlled and safe alternative.

Keywords: hysterectomy, transversus abdominis plane block, paravertebral block, postoperative pain

\section{Introduction}

In many patients undergoing abdominal surgery, epidural analgesia remains the standard for provision of postoperative analgesia. However, there are a number of clinical situations where epidural analgesia is contraindicated, is refused, or fails. Moreover, many operations do not necessitate the postoperative use of continuous epidural analgesia, and pain relief in these patients may be successfully achieved using other methods. 
In many Norwegian hospitals, hysterectomies are performed under general anesthesia without the use of epidural blockade. Postoperative analgesia in these patients is often provided using patient-controlled analgesia (PCA) with morphine or other opioids. Additionally, these women receive paracetamol on a regular basis, and occasionally nonsteroidal anti-inflammatory drugs. In our institution (the Norwegian Radium Hospital - Oslo University Hospital, Oslo, Norway), we perform about 400 hysterectomies a year due to gynecological cancer. The vast majority of these operations include not only the removal of the uterus but also bilateral salpingo-oophorectomy and omentectomy, and some of these patients may also require pelvic and/or paraaortal lymph node dissection. Postoperatively, many of these patients do not receive epidural analgesia, but a standardized analgesic regimen consisting of regular intravenous (IV)/ oral paracetamol $1 \mathrm{~g}$ every 6 hours combined with PCA ketobemidon (bolus $1 \mathrm{mg}$, lockout 7 minutes, 1 hour maximum dose $8 \mathrm{mg}$ ). Ketorolac IV injections 10-30 mg are used in some patients who require additional analgesia.

The transversus abdominis plane (TAP) block is a novel method of regional anesthesia that provides unilateral anesthesia to the anteriolateral abdominal wall. A bilateral technique has also been used in various clinical situations. ${ }^{1-4}$ Recent studies have demonstrated that bilateral TAP block in combination with general anesthesia provides sufficient and long-lasting postoperative pain relief after abdominal surgery, thus significantly reducing the requirements for opioids, and presumably opioid-induced side effects. ${ }^{1-4}$ In patients undergoing hysterectomy, Carney et $\mathrm{al}^{4}$ have demonstrated that bilateral TAP block with ropivacaine in combination with general anesthesia provides effective postoperative analgesia and significantly reduces postoperative cumulative morphine consumption postoperatively for up to 48 hours. There were no complications attributable to the TAP block. All TAP patients reported high levels of satisfaction.

Similarly, thoracic paravertebral block (PVB) has been demonstrated to provide effective postoperative analgesia in patients undergoing minor and major abdominal surgery by blocking sensory innervation of the abdominal wall. PVB significantly reduces the need for supplemental opioid administration, reduces postoperative nausea and vomiting (PONV), and in some patient groups shortens hospital stay. .-7 $^{5-1}$ Although PVB has been demonstrated to be effective in patients undergoing abdominal and thoracic surgery, ${ }^{7-10}$ its analgesic efficacy after major gynecological surgery has not, as yet, been reported.
Therefore, the objective of the present study was to evaluate the analgesic properties of bilateral thoracic PVB in patients undergoing hysterectomy and to compare its effectiveness with that of bilateral TAP block. We also hypothesize that due to extensive surgery performed via a midline vertical incision, and, as a result, increased stimulation of the pelvic and abdominal visceral sites, our patients experience more pain postoperatively than those who undergo a conventional hysterectomy with a Pfannenstiel incision. As such, the second intention of the present study was to investigate whether a bilateral TAP block in combination with general anesthesia would be as effective in this setting as it was in the study of Carney et al. ${ }^{4}$

\section{Methods}

The study protocol was approved by the Regional Ethics Committee.

Between March and November 2010, 60 patients scheduled for elective abdominal hysterectomy at the Norwegian Radium Hospital (Oslo University Hospital) with bilateral salpingo-oophorectomy were recruited, and a written consent was obtained from each. Patients with a history of allergic reaction to local anesthetics and on chronic pain medications (both opioids and nonopioids) were not included.

After having signed the consent letter, each patient was immediately allocated to a study group by using the method of random numbers. These numbers were generated by a computer program (http://www.randomizer.org), and three treatment groups were created. Two first authors enrolled the participants and performed all the blocks.

Two patient groups received either a bilateral PVB $(\mathrm{n}=19)$ or a TAP block $(\mathrm{n}=19)$, while a third group of 20 served as a control. Patients in this group received saline injections paravertebrally $(\mathrm{n}=10)$ or in the TAP $(\mathrm{n}=10)$. The volume of saline delivered to each group was identical to that suggested for each individual block.

TAP blocks were placed under dynamic ultrasound guidance (S-Nerve, SonoSite Inc, Bothell, WA) after the induction of general anesthesia. Following skin preparation, a linear probe was placed in an axial plane between the costal margin and the iliac crest. After identifying the three muscular layers of the abdominal wall, the tip of the $18 \mathrm{G}$ Tuohy needle was inserted between the internal oblique and the TAP muscles using the in-plane technique. Patients received $0.375 \mathrm{~mL} / \mathrm{kg} /$ side of $0.25 \%$ bupivacaine with epinephrine $5 \mu \mathrm{g} / \mathrm{mL}$, yielding a final dose of bupivacaine of $1.875 \mathrm{mg} / \mathrm{kg}$. 
The PVB was performed prior to induction of general anesthesia. Single injections were placed at the level of T10. The patient was placed in the sitting position, and the spinal process of the T10 was palpated. Then, with the use of the ultrasound scanner (S-Nerve, SonoSite Inc), the corresponding transverse process was visualized, and the skin-transverse process distances were measured on both sides. After infiltration of skin and subcutaneous tissue with $1 \%$ lidocaine with epinephrine $5 \mu \mathrm{g} / \mathrm{mL}$, an $18-\mathrm{G}$ Tuohy needle was inserted perpendicular to the skin, approximately $2.5 \mathrm{~cm}$ from the midline, until contact with the transverse process was established. The needle was then slightly withdrawn and reinserted $1-1.5 \mathrm{~cm}$ deeper either caudally or cranially for the transverse process. After careful aspiration, patients received a slow injection of $0.25 \mathrm{~mL} / \mathrm{kg} / \mathrm{side}$ of $0.375 \%$ bupivacaine with epinephrine $5 \mu \mathrm{g} / \mathrm{mL}$, yielding the same dose of bupivacaine of $1.875 \mathrm{mg} / \mathrm{mL}$.

All study patients received standard pre- and intraoperative monitoring. All patients received standard premedication with oral oxazepam. General anesthesia was induced with fentanyl $(2-4 \mu \mathrm{g} / \mathrm{kg})$ and thiopental $(3-5 \mathrm{mg} / \mathrm{kg})$, and tracheal intubation was facilitated with cis-atracurium $(0.15-0.2 \mathrm{mg} / \mathrm{kg})$. All patients were artificially ventilated, and maintenance of anesthesia was achieved by the inhalation of sevoflurane/air/ $\mathrm{O}_{2}$ mixture and incremental doses of fentanyl (50-100 $\mu \mathrm{g}$ ). The end-tidal concentration of sevoflurane was adjusted to $0.8-1.0$ minimal alveolar concentration. Incremental bolus doses of ephedrine were used to control hypotension. No prophylactic antiemetics were given.

After the surgery and extubation, the patients were transferred to the recovery room, and a standardized analgesic regimen consisting of regular IV/oral paracetamol $1 \mathrm{~g}$ every 6 hours combined with PCA ketobemidon (bolus $1 \mathrm{mg}$, lockout 7 minutes, 1 hour maximum dose $8 \mathrm{mg}$ ) was initiated. Patients requiring additional analgesia received IV injections of ketorolac (15-30 mg). The severity of pain and cumulative ketobemidon consumption was assessed by an independent investigator blinded for the study protocol. Pain intensity was assessed using a numeric rating scale (NRS) $(0=$ no pain, $10=$ worst imaginable pain). All patients were assessed at rest and on coughing. PONV were assessed using a categorical scoring $\operatorname{system}(0=$ none, $1=$ mild, $2=$ moderate, $3=$ severe $)$, and antiemetics were offered to all patients complaining of PONV. These assessments were performed at 2, 4, 6, 24, and 48 hours after completion of surgery. Additionally, 48 hours following surgery, patients were asked to report their satisfaction with the postoperative analgesia regimen using a categorical scoring system $(1=$ satisfied, 2 = cannot answer, 3 = dissatisfied).

The primary endpoint of our study was cumulative ketobemidone consumption 24 hours after the surgery. We estimated our sample size on the basis of the results of a similar study performed by McDonnell et al. ${ }^{2}$ In this study, the authors compared the efficacy of TAP block with placebo in patients undergoing abdominal surgery via a midline incision. Based on their pilot data, they calculated that a clinically important reduction in postoperative morphine consumption would require only 14 patients in each group, projecting a 24-hour morphine requirement of $60 \mathrm{mg}$ with a standard deviation of $\pm 10 \mathrm{mg}$, using $\alpha=0.05$ and $\beta=0.2$. They confirmed this hypothesis, demonstrating that 16 patients were sufficient to demonstrate a $70 \%$ reduction in morphine consumption 24 hours following surgery. At the moment, there is no information about the analgesic efficacy of thoracic PVB in gynecological surgery. We, however, hypothesized that PVB would be at least as efficient as TAP in providing postoperative analgesic relief. To minimize any effect of data loss we determined to include 20 patients in every study group. The secondary endpoint of the study was pain intensity 48 hours after the surgery.

\section{Calculation and statistics}

Values throughout this report are indicated as mean \pm standard deviation. Statistical analyses were performed using a standard statistical program (SPSS PASW Statistics 18; IBM Corporation, Armonk, NY). Demographic data were analyzed using Kruskal-Wallis and Mann-Whitney tests.

The group and time variables were defined as the between-subjects and within-subjects factors. Repeated measures analysis of variance using a mixed model approach and assuming compound symmetry structure for the variancecovariance matrix were used to test for difference between the three groups (pain scores and ketobemidon consumption), and the pairwise comparisons for each time point were performed with the Bonferroni correction. The goodness of fit was examined by plotting a histogram of residuals for overall and for each time point. In all cases, a $P$-value of 0.05 or less was considered statistically significant.

\section{Results}

Demographic data are presented in Table 1. There were no statistically significant differences between the groups in terms of age, height, weight, duration of surgery, and intraoperative use of ephedrine. Total fentanyl dose was, 
Table I The perioperative data ${ }^{\mathrm{a}}$

\begin{tabular}{|c|c|c|c|}
\hline & $\begin{array}{l}\text { PVB and PCA } \\
(n=19)\end{array}$ & $\begin{array}{l}\text { TAP and PCA } \\
(n=19)\end{array}$ & $\begin{array}{l}\text { Control (PCA) } \\
(n=20)\end{array}$ \\
\hline Age (years) & $60.4 \pm 9.6$ & $60.4 \pm 9.8$ & $59.9 \pm 10.8$ \\
\hline Height $(\mathrm{cm})$ & $164.4 \pm 5.5$ & $165.7 \pm 7.5$ & $166.1 \pm 5.3$ \\
\hline Weight (kg) & $68.1 \pm 13.4$ & $70.2 \pm 12.9$ & $73.8 \pm 16.1$ \\
\hline ASA (I/II/III) (n) & $3 / 15 / 1$ & $2 / 16 / 1$ & $3 / 15 / 2$ \\
\hline Duration of surgery $(\mathrm{min})$ & $143 \pm 50$ & $|5| \pm 49.2$ & $134 \pm 46.3$ \\
\hline \multicolumn{4}{|l|}{ Type of surgery } \\
\hline Hysterectomy and bilateral salpingo-oophorectomy alone or with & 4 & 6 & 7 \\
\hline O/LND/O + LND (n) & $0 / 4 / 11$ & $2 / 6 / 5$ & $2 / 6 / 5$ \\
\hline Total fentanyl dose $(\mu g)$ & $344 \pm 97.3^{b}$ & $415 \pm 111.9$ & $425 \pm 81.9$ \\
\hline Total ephedrine dose (mg) & $7.6 \pm 7.7$ & $5 \pm 6.9$ & $4.5 \pm 5.6$ \\
\hline
\end{tabular}

Notes: ${ }^{\vee}$ Values are presented as numbers and mean \pm standard deviation; bintraoperative fentanyl dose was significantly lower in the PVB group compared with both the TAP group and the control group $(P<0.05)$.

Abbreviations: ASA, American Society of Anesthesiologists; LND, lymph node dissection; O, omentectomy; PCA, patient-controlled analgesia; PVB, paravertebral block; TAP, transversus abdominis plane.

however, significantly lower in the PVB group compared with both the control and the TAP block groups.

Two of 60 recruited patients were later excluded from the study protocol for the following reasons. In one patient assigned to the TAP block group, the procedure was impossible to perform due to the patient being grossly obese. The second patient assigned to the PVB group was excluded because of poorly controlled hypothyroidism and analgesic protocol violations (see Figure 1).
TAP block patients required significantly less ketobemidon at 6 hours postoperatively compared with the control group. During the late postoperative period (at 24 hours and 48 hours), cumulative ketobemidon consumption was significantly reduced in both block groups compared with in the control group (Figure 2). Ketobemidon consumption at these time intervals was significantly lower in the PVB group compared with in the TAP block group (Figure 2).

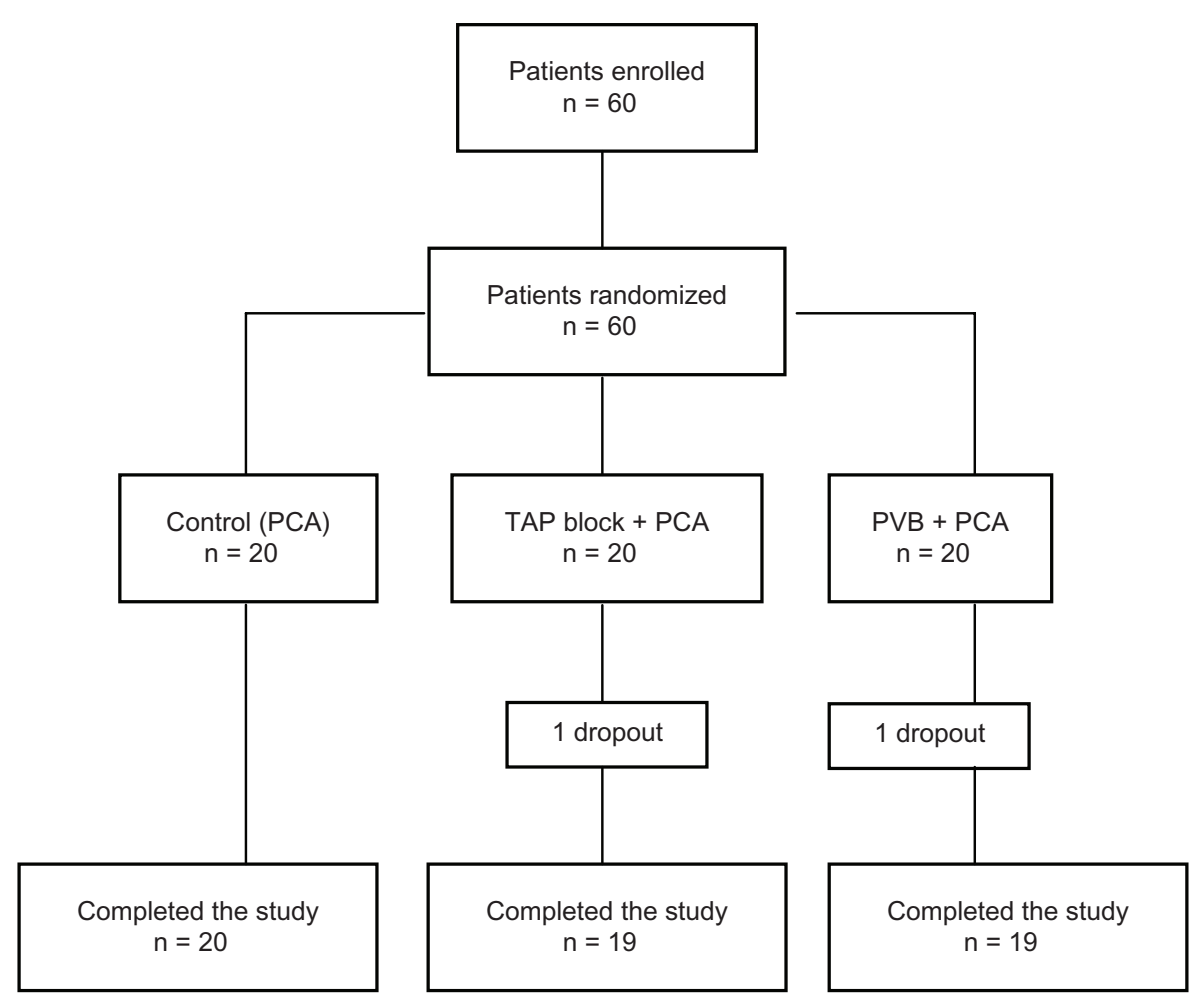

Figure I Patient flow diagram throughout the study.

Abbreviations: PCA, patient-controlled analgesia; PVB, paravertebral block; TAP, transversus abdominis plane. 
Postoperative ketobemidon consumption (mg)

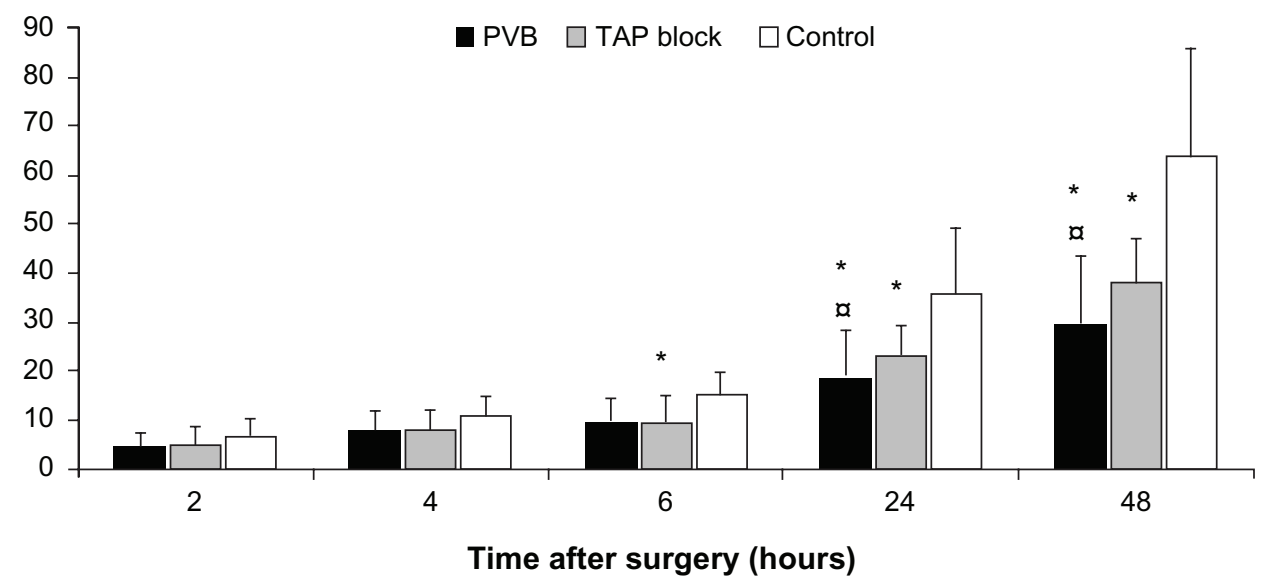

Figure 2 Mean postoperative cumulative ketobemidon consumption.

Notes: *Significant difference between the treated group and the control group $(P<0.05)$; "significant difference between the PVB group and the TAP block group $(P<0.05)$.

Abbreviations: PVB, paravertebral block; TAP, transversus abdominis plane.

Both PVB and transversus abdomins block significantly reduced pain scores at all time intervals, except in the TAP block group 48 hours following surgery (Figure 3). Postoperative pain intensity on coughing was also significantly reduced by both forms of block compared with placebo, while PVB was significantly more effective than the TAP block at 2 hours (Figure 4). One patient in the control group received a rescue injection of ketorolac (30 mg IV) during her stay in the recovery room.

PONV scores were low in all groups, but during the early postoperative period (6 hours) more control group patients needed antiemetics compared with the treatment groups (Table 2).

Both methods of block provided a sufficient degree of patient satisfaction, while only $50 \%$ of patients in the control group indicated that they were satisfied with the postoperative level of analgesia (Table 3). Neither complications nor unintended effects were registered throughout the course of the study.

\section{Discussion}

Our study demonstrates the analgesic effectiveness of both PVB and TAP block after major gynecological cancer surgery.

Pain intensity (NRS)

- PBV $\square$ TAP block $\square$ Control

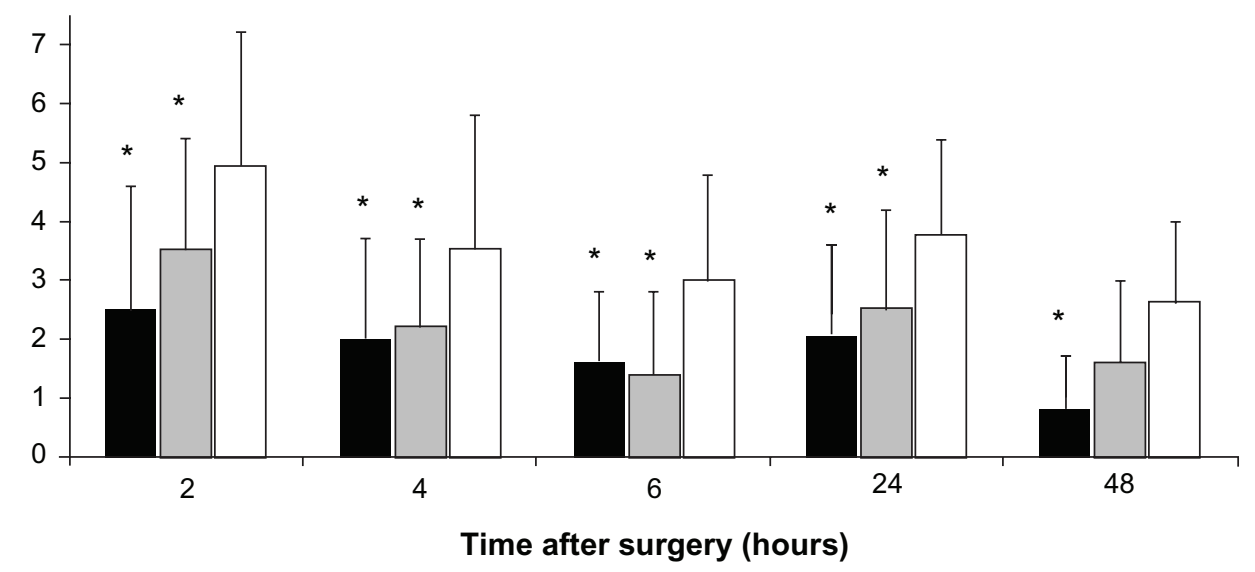

Figure 3 Mean postoperative NRS scores at rest.

Note: $*$ Significant difference between the treated group and the control group $(P<0.05)$.

Abbreviations: NRS, numeric rating scale; PVB, paravertebral block; TAP, transversus abdominis plane. 


\section{Pain intensity (NRS) on coughing}

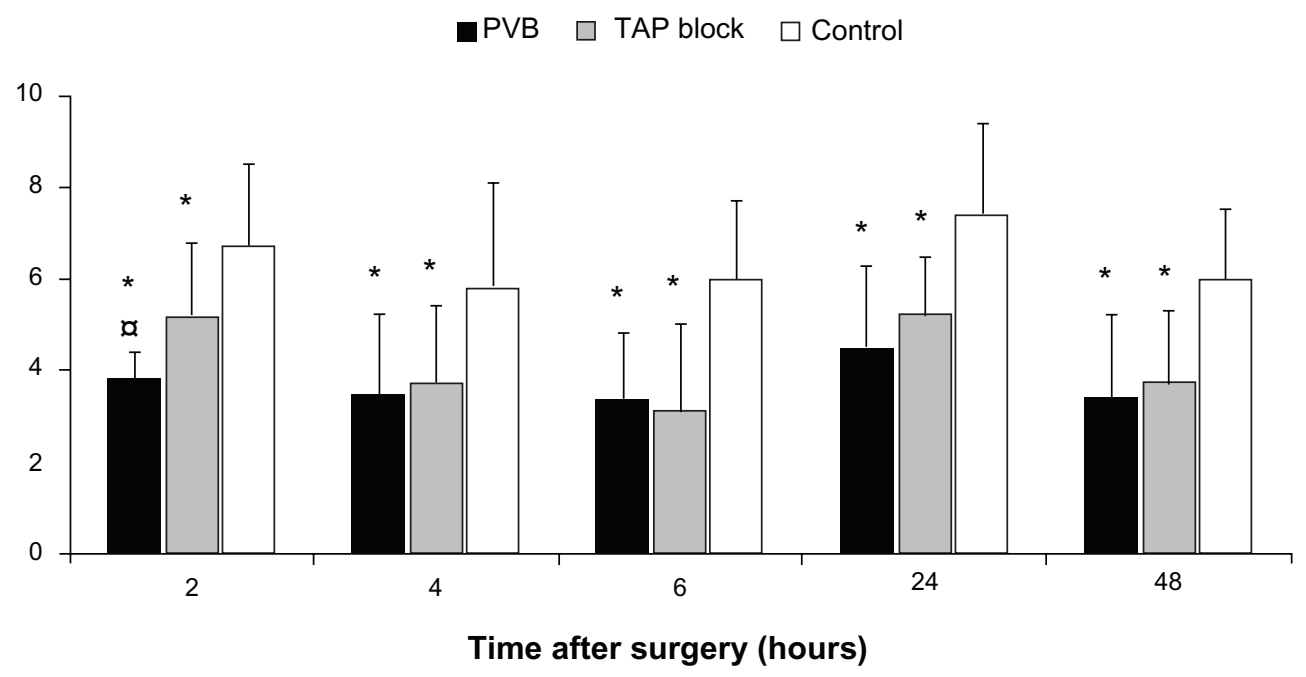

Figure 4 Mean postoperative NRS scores on coughing.

Notes: *Significant difference between the treated group and the control group $(P<0.05)$; "significant difference between the PVB group and the TAP block group $(P<0.05)$.

Abbreviations: NRS, numeric rating scale; PVB, paravertebral block; TAP, transversus abdominis plane.

Both regional anesthetic techniques demonstrated significant reductions in both opioid requirements and pain scores during early and late postoperative periods. Our results support previous findings that TAP block performed preoperatively provides sufficient and long-lasting analgesia. ${ }^{2-4,11}$ They also specifically support the results reported in Carney et al's study ${ }^{4}$, that TAP block has been demonstrated to be effective in patients undergoing abdominal hysterectomy. Moreover, our findings demonstrate the postoperative analgesic efficacy of TAP block in women undergoing hysterectomy via a midline abdominal incision. PONV scores were low in all groups, but during the early postoperative period more control group patients needed antiemetics. This observation may be explained by differences in intraoperative fentanyl dose between the study groups.

Previously, PVB has been demonstrated to be effective in breast, hernia, and thoracic surgery; ${ }^{5,10,12}$ however, evidence of its effectiveness in gynecological surgery has been limited

Table 2 Use of antiemetics during early (6 hour) postoperative period

\begin{tabular}{llll}
\hline & PVB & TAP block & $\begin{array}{l}\text { Control } \\
\mathbf{n}=\mathbf{2 0}\end{array}$ \\
\hline I med & 2 & $\mathbf{n}=\mathbf{1 9}$ & 2 \\
2 meds & 2 & 3 & 7 \\
3 meds & & 5 & \\
4 meds & & & I \\
Total & 4 patients & 8 patients & 10 patients \\
\hline
\end{tabular}

Abbreviations: PVB, paravertebral block; TAP, transversus abdominis plane. to only one study from $1955 .{ }^{13}$ Thus, our findings appear to be the first to systematically demonstrate the efficacy of this form of block in major gynecological surgery.

Local anesthetics injected paravertebrally exhibit their effects by blocking spinal nerves either in the paravertebral space or due to intercostal and/or epidural spread. The degree of epidural spread after thoracic paravertebral injections is highly variable; up to $70 \%$ of injectate is reported to reach the epidural space in patients ( $40 \%$ in cadavers) $)^{14,15}$ As such, thoracic PVB may be considered as a paraneuroaxial block, and one might expect the development of significant arterial hypotension. Therefore, in our study we did not use the concentrated solutions for PVBs and performed the injections slowly, because high-pressure injections have been demonstrated to increase the propensity toward unwanted epidural spread. ${ }^{16}$ All our patients remained hemodynamically stable during the perioperative period, and the mean intraoperative dose of ephedrine used to control the hypotension did not statistically differ between the PVB and the control group (7.6 $\pm 1.8 \mathrm{mg}$ vs $4.5 \pm 1.3 \mathrm{mg}$ ). Nevertheless, the potential

Table 3 Satisfaction with the postoperative analgesia regimen

\begin{tabular}{llll}
\hline & PVB & TAP block & Control \\
& $\mathbf{n = 1 9}$ & $\mathbf{n = 1 9}$ & $\mathbf{n = 2 0}$ \\
\hline Satisfied $(\mathrm{n})$ & 16 & 15 & 10 \\
Cannot answer $(\mathrm{n})$ & 3 & 3 & 7 \\
Dissatisfied $(\mathrm{n})$ & & $\mathrm{I}$ & 3 \\
\hline
\end{tabular}

Abbreviations: PVB, paravertebral block; TAP, transversus abdominis plane. 
limitation of our study is its limited size, which does permit assessment of the safety of bilateral PVB.

Another limitation of this study is that we did not assess the success rate of each form of block. Thus, it is possible that some patients in the study groups had unilateral, patchy, or even failed blocks. As such, in the ideal situation where a $100 \%$ success rate was achieved, the results of the study might have been somewhat different. It, however, would only have increased the effectiveness of the blocks. While designing the protocol we intended to study both the height and the success rate of the blocks. However, our recovery personnel who were supposed to blindly register these data did not entirely understand their task and failed to collect the data correctly. This mistake was discovered only after the completion of the study when we had already engaged in data analysis.

The third study limitation is the fact that the sensory block of the skin would perhaps reduce the blinding of the patients during the postoperative period, although we truly believe that they were sufficiently blinded to the study protocol.

We conclude that both TAP block and PVB may be used as effective analgesia adjuncts in women undergoing major gynecological surgery where epidural analgesia is not indicated or desired. Both forms of block provided additional analgesia and reduced the amount of systemic opioids administered and opioid-induced side effects, and each might be expected to facilitate postoperative recovery. The fact that only $50 \%$ of patients in the PCA group in our study were satisfied with the level of postoperative analgesia can also be viewed as an argument in favor of the inclusion of a regional block into a multimodal analgesia regimen. Although in our study thoracic PVB appeared to be more effective than transversus abdominis block, the latter performed under ultrasound guidance seems to be more controlled and a safe alternative.

\section{Acknowledgments}

The authors thank Toril AW Gaardsrud and Tori Fossum Sorgendal for technical assistance in the performance of the study described, and Professor David A Lathrop for reading the manuscript and offering suggestions for changes.

\section{Disclosure}

Presented as a poster at the World Congress of Anesthesiologists, Buenos Aires, Argentina, 2012. The authors report no conflicts of interest in this work.

\section{References}

1. O'Donnell BD, McDonnell JG, McShane AJ. The transversus abdominis plane (TAP) block in open retropubic prostatectomy. Reg Anesth Pain Med. 2006;31:91.

2. McDonnell JG, O’Donnell B, Curley G, Heffernan A, Power C, Laffey JG The analgesic efficacy of transversus abdominis plane block after abdominal surgery: a prospective randomized controlled trial. Anesth Analg. 2007;104:193-197.

3. McDonnell JG, Curley G, Carney J, et al. The analgesic efficacy of transversus abdominis plane block after cesarean delivery: a randomized controlled trial. Anesth Analg. 2008;106:186-191.

4. Carney J, McDonnell JG, Ochana A, Bhinder R, Laffey JG. The transversus abdominis plane block provides effective postoperative analgesia in patients undergoing total abdominal hysterectomy. Anesth Analg. 2008;107:2056-2060.

5. Naja Z, Ziade MF, Lonnqvist PA. Bilateral paravertebral somatic nerve block for ventral hernia repair. Eur J Anaesthesiol. 2002;19:197-202.

6. Klein S, Pietrobon R, Nielsen K, et al. Paravertebral somatic nerve block compared with peripheral nerve blocks for outpatient inguinal herniorrhaphy. Reg Anesth Pain Med. 2002;27:476-480.

7. Richardson J, Vowden P, Sabanathan S. Bilateral paravertebral analgesia for major vascular surgery: a preliminary report. Anaesthesia. 1995;50:995-998.

8. Eason MJ, Wyatt R. Paravertebral thoracic block: a reappraisal. Anaesthesia. 1979;34:638-642.

9. Richardson J, Sabanathan S. Thoracic paravertebral analgesia. Acta Anaesthesiol Scand. 1995;39:1005-1015.

10. Joshi G, Bonnet F, Shah R, et al. A systematic review of randomized trials evaluating regional techniques for posthoracotomy analgesia. Anesth Analg. 2008;107:1026-1040.

11. Siddiqui MR, Sajid MS, Uncles DR, Cheek L, Baig MK. A metaanalysis on the clinical effectives of transversus abdominis plane block. J Clin Anesth. 2011;23:7-14.

12. Schnabel A, Reichl SU, Kranke P, Pogatzki-Zahn EM, Zahn PK. Efficacy and safety of paravertebral blocks in breast surgery: a meta-analysis of randomized controlled trials. Br J Anaesth. 2010;105:842-852.

13. Siliquini PN, Revelli E. Benefits and effects of paravertebral infiltration of long-acting procaine in obstetrics and gynecology. Minerva Ginecol. 1955;15:7:163-169.

14. Purcell-Jones G, Pither CE, Justins DM. Paravertebral somatic nerve block: a clinical, radiographic, and computed tomographic study in chronic pain patients. Anesth Analg. 1989;68:32-39.

15. Cowie B, McGlade D, Ivanusic J, Barrington MJ. Ultrasound-guided thoracic paravertebral blockade: a cadaveric study. Anesth Analg. 2010; 110:1735-1739.

16. Gadsden JC, Lindenmuth DM, Hadzic A, Xu D, Somasundarum L, Flisinski KA. Lumbar plexus block using high-pressure injection leads to contralateral and epidural spread. Anesthesiology. 2008;109: 683-688.
Local and Regional Anesthesia

\section{Publish your work in this journal}

Local and Regional Anesthesia is an international, peer-reviewed, open access journal publishing on the development, pharmacology, delivery and targeting and clinical use of local and regional anesthetics and analgesics. The journal welcomes submitted papers covering original research, basic science, clinical studies, reviews \& evaluations,

\section{Dovepress}

guidelines, expert opinion and commentary, case reports and extended reports. The manuscript management system is completely online and includes a very quick and fair peer-review system, which is all easy to use. Visit http://www.dovepress.com/testimonials.php to read real quotes from published authors. 